\title{
Efficacy of hand held, inexpensive UV light sources on Acanthamoeba, causative organism in amoebic keratitis
}

This article was published in the following Dove Press journal:

Clinical Optometry

20 January 2010

Number of times this article has been viewed

\author{
Ivan Cometa' \\ Andrew Rogerson' \\ Scott Schatz ${ }^{2}$ \\ 'Department of Biology, California \\ State University Fresno, Fresno, CA, \\ USA; ${ }^{2}$ Arizona College of Optometry, \\ Midwestern University, Glendale, \\ AZ, USA
}

\begin{abstract}
Multipurpose lens cleaning solutions (MPS) fail to consistently kill or inactivate Acanthamoeba cysts and UV irradiation, while effective at high doses, can damage contact lenses. The present study considered synergy of action between MPS and hand-held inexpensive (ie, relatively weak) UV irradiation units. Regardless of disinfection method recently formed cysts ( $<10$ days) were far more susceptible to treatment than mature cysts ( $>14$ days). This has important implications for future protocols on testing methods for killing amoebae. The study also showed that cysts of different strains (two tested, FLA2 and P120) are variable in their response to MPS, presumably reflecting differences in cyst wall structure and thus permeability to the disinfectant. On the other hand, the effect of UV irradiation was not wall structure dependent. A 6-hour treatment with MPS alone killed trophic amoebae but failed to kill any mature cysts. Cysts of strain FLA2 were killed after 24 hours with MPS but cysts of strain P120 survived. UV irradiation with the larger $4 \mathrm{~W}$ unit killed all cysts after 7 minutes and was more effective than the smaller battery-powered unit (after 10 minutes about $50 \%$ of cysts were killed). When the larger unit was used with the MPS disinfection, all trophozoites were killed using UV for 3 minutes and MPS for 1 hour. The resistant P120 cysts remained a challenge but a 2- to 4-minute UV treatment followed by MPS for 3 or 6 hours reduced mature cyst survival by about $50 \%$. The small unit in combination with MPS was less effective but did reduce the time required to kill trophic amoebae in MPS (6 hours MPS alone versus 3 hours MPS with a 1-minute UV treatment). In short, inexpensive UV units do enhance MPS disinfection and future lens cleaning systems/protocols might capitalize on this synergistic action.
\end{abstract}

Keywords: UV light sources, amoebic keratitis, MPS

\section{Introduction}

In recent years, a number of studies have investigated the efficacy of various multi-purpose and hydrogen peroxide based contact lens disinfection solutions against species of Acanthamoeba. ${ }^{1,2}$ Species of Acanthamoeba are considered by Page ${ }^{3}$ to be possibly the most common free-living protozoa in nature and they are ubiquitous in soil, freshwater and marine systems. ${ }^{4}$ These amobae are of interest to health care providers in general and eye care providers in particular because of their opportunistic association with sight-threatening corneal infections. The life history of Acanthamoeba includes a vegetative trophozoite stage and a thick-walled resting cyst stage. Speciation was originally based on morphological characteristics but current classification schemes are now based, in part, on molecular markers which have led to the creation of up to 14 molecular types (genotypes). Interestingly, even though genotype 4 (T4) is most commonly associated with acanthamoebic keratitis (AK), there is no known
Correspondence: Andrew Rogerson Department of Biology, California State University Fresno, Fresno, CA 93740, USA Email arogerson@csufresno.edu 
character which confers virulence and a higher likelihood to cause AK. The incidence of AK is largely within the contact lens wearing population, with about $85 \%$ of cases occurring in contact lens wearers and at a rate of about 1 to 2 cases per million contact lens wearers. The primary issue regarding AK is that, even though the incidence is rare, the morbidity factor is high. In view of recent outbreaks of AK associated with poor contact lens hygiene and, coupled with the inability of most MPS to kill the amoebae, we undertook a study to examine the efficacy of a combination of UV irradiation (from inexpensive hand-held units) and exposure to a MPS solution.

\section{Materials and methods}

\section{Culture methods for Acanthamoeba}

\section{and source of strains}

Two Acanthamoeba strains from a collection of strains held by one of the authors (AR) were used in this study. All cultures were from known sources and have been previously genotyped. ${ }^{4}$ The strains used were FLA2, a T4 genotype isolated from beach sand in Southern Florida and strain P120, a T3 genotype isolate from tapwater in Hong Kong. Amoebae were routinely cultured on non-nutrient agar (NNA) made with amoeba saline. ${ }^{3}$ These agar plates were streaked with Escherichia coli as a food source. A block of agar containing exponentially growing cells was dissected from the plate and placed amoeba side down on a dense, fresh E. coli streak. Amoebae consumed the bacterial prey, reproduced and migrated along the streak. Trophic amoebae were evident at the advancing edge and cysts formed as prey were exhausted. This allowed blocks to be chosen that comprised of trophic amoebae (trophozoites) or cysts. This culture method provided ample cells for experimentation. A healthy growing culture yielded up to several million cells on a single agar plate (pers comm AR). Stock cultures were transferred every 2 weeks to fresh plates.

For the experiments, exponentially growing trophozoites were sampled from the advancing edge of a recently sub-cultured plate. For treatments with cysts, 'young' cysts were formed less than 10 days after sub-culture and 'mature' cysts were older than 14 days. Cyst age was found to be an important variable in susceptibility to treatment.

\section{Testing methods}

Effectiveness of disinfection against acanthamoebae was tested using a multipurpose cleaning solution (MPS) alone and in combination with two inexpensive UV irradiation devices. The MPS was the popular product OptiFree ${ }^{\circledR}$ Express (Alcon, Ft. Worth, TX, USA). During testing, only fresh MPS was used; an open bottle was never more than 7 days from time of purchase.
Two inexpensive UV sources were chosen since the goal of this work was to determine whether relatively weak UV irradiation could supplement disinfection with MPS. The first unit was a mini UV battery powered lamp (model UVG4, manufactured by UVP, Upland, CA, USA) with a shortwave tube (254 nm, UV-C irradiation) operated at $4 \mathrm{~W}$ power (cost less than US\$50). This unit is referred to as the small lamp. The second unit was more powerful and was 120 $\mathrm{V}$ rather than $12 \mathrm{~V}$. This unit was the Spectroline E-Series hand lamp manufactured by Spectronics Corp., Westbury, NY, USA. The unit cost around US\$150. This unit generated UV-C irradiation at $254 \mathrm{~nm}$. This unit is referred to as the large lamp. In both cases, since UV is harmful to both eyes and skin, protective gloves and glasses were used.

Regardless of the disinfection method tested, the test procedure was the same in all cases. Acanthamoeba were grown on agar plates with streaks of $E$. coli as bacterial prey. For testing trophic amoebae, agar blocks (about $10 \mathrm{~mm} \times$ $10 \mathrm{~mm}$ ) containing approximately 50 cells were cut from a recently inoculated agar plate (within 4 days). Trophozoites were evident at the advancing edge of the amoeba population. As prey was exhausted acanthamoebae encysted. Picking up agar blocks containing approximately 50 cysts allowed for testing with both young and mature cysts. As noted by Shoff et $\mathrm{al}^{2}$ testing the effectiveness of amoebae on agar has two distinct advantages. It allows for the unambiguous selection of cysts or trophozoites (these are evident when blocks are selected under the dissecting microscope, $\times 40)$ and the method mimicks the response of cells attached to a surface. Agar blocks containing amoebae were transferred to the wells of a 24-well tissue culture plate made of untreated polystyrene (Corning Incorporated, Corning, NY, USA). Care was taken to ensure that amoebae were on the uppermost face of the blocks. Wells contained either $1 \mathrm{~mL}$ of amoeba saline ${ }^{3}$ or MPS. In all treatments, a grow-out control used a single agar block with cells in amoeba saline. After treatment (MPS, UV, or MPS/UV) agar blocks with amoebae were transferred to Difco Dey/Engley Broth (Becton, Dickinson and Co., Sparks, MD, USA) to neutralize any disinfectant. This step has been shown to have no effect on amoebae ${ }^{5}$ and control blocks, also subject to this neutralization step, were always positive for amoebae. After neutralizing for 5 minutes and rinsing twice in amoeba saline ( 5 minutes and 10 minutes each), blocks were placed amoeba side down on a fresh NNA plate streaked with $E$. coli to stimulate the growth of any surviving amoebae. Plates were sealed in Parafilm and incubated at room temperature (about $22^{\circ} \mathrm{C}$ ). The distance migrated by surviving and growing amoebae was noted each 
day using a dissecting microscope at $40 \times$ magnification. It is important to note that this method allowed for the rapid screening of treatments that caused $100 \%$ mortality. It did not give information on the number of cells killed in treatment unless all 50 cells on an agar block were inactivated.

\section{UV treatment alone}

Agar blocks containing trophozoites or cysts (of two strains, FLA 2 and P120) were added to amoeba saline in the wells of a 24-well culture plate. Saline was added until amoebae were about $0.5 \mathrm{~mm}$ below the water surface. Lamps were rested on the top of the culture plate $17 \mathrm{~mm}$ above the amoebae. This mimicked conditions that might exist in a lens case containing a contact lens. Both the small and large lamps were tested using treatment times of 1, 2, 3, 4, 7 or 10 minutes. Controls were run in amoeba saline without UV treatment. In all cases, either 6 or 10 replicate agar blocks were used.

\section{MPS treatment alone}

Agar blocks containing trophozoites or cysts (FLA 2 and P120) were added to OptiFree ${ }^{\circledR}$ cleaning solution for 1, 3, 6 or 24 hours of treatment. Controls were run in amoeba saline. In all cases, either 6 or 10 replicate agar blocks were used.

\section{Combined treatment, UV and MPS}

This treatment used both trophic amoebae and cysts from two strains (FLA2 and P120) and incorporated irradiation from both the small and large UV lamps. Agar blocks containing amoebae were transferred to wells containing $1 \mathrm{~mL}$ of MPS. At this time, amoebae were irradiated with UV light (1, 2, 3 and 4 minutes). It was reasoned that treating the cells with UV at the start of the MPS cleaning would weaken Acanthamoeba and make them more susceptible to chemical disinfection. After UV treatment, blocks were left for 1, 3, 6 or 24 hours in MPS. Controls were run in amoeba saline without UV treatment. In all cases, either 6 or 10 replicate agar blocks were used.

\section{Results}

Cyst age has been reported to be an important variable affecting the outcomes of disinfection. ${ }^{6}$ To confirm or refute this claim, an initial set of experiments compared the effect of MPS disinfection and UV irradiation on young $(<10$ days $)$ and mature $(>14$ days $)$ cysts. Additional trials with slightly older cysts up to 18 days showed no difference beyond 14 days cysts In general, recently formed cysts were more susceptible to treatment (MPS or UV) than mature cysts (Table 1). This was most evident in the case of MPS disinfection. A 6 and 24 hours MPS treatment (without UV) killed or inactivated all the young cysts of strain FLA2 and reduced the populations significantly in the case of the P120 strain. However, with the exception of strain FLA2 after 24 hours mature cysts were unaffected. The same data clearly show strain differences; the T4 genotype (FLA2) was far more susceptible to treatment than the T3 genotype (P120). The data on effectiveness of the handheld UV systems is less clear-cut in this initial experiment but clearly the larger UV bulb was more effective than the smaller unit that failed to kill all the cysts on an agar block (approximately 50 cysts per replicate). All experiments thereafter used mature cysts since these were more resistant to treatment and afforded a more rigorous challenge.

The effect of UV irradiation alone is summarized in Table 2 . Here, the large unit was clearly more effective than the small UV source in terms of inactivating both cysts and trophozoites. After 3 minutes of treatment with the large UV bulb, both trophozoites and cysts were reduced by about $50 \%$ regardless of strain. It is interesting to note that strain susceptibility to treatment, evident in the case of MPS treatment, was not evident in these UV trials. Trophozoites of both strains were killed after 4 minutes treatment and cysts after 7 minutes. The small unit was far less effective although after 10 minutes of treatment, survival of both cysts and trophozoites was reduced by approximately $50 \%$.

The effect of the MPS (OptiFree ${ }^{\circledR}$ Express) alone is shown in Table 3. As also shown in Table 1, the cysts of the T4 genotype (FLA2) were more susceptible to treatment than the T3 genotype. After 24 hours treatment, all cysts of FLA2 were inactivated (in all, some 500 cysts $[50$ cysts/block $\times 10$ agar blocks] were challenged in these agar block replicates) while

Table I Influence of cyst age (young [ $<10$ days] versus mature [ $>$ I 4 days]) on the effectiveness of treatment by UV irradiation for 4 minutes

\begin{tabular}{llll}
\hline Treatment & Strain & Immature cysts & Mature cysts \\
\hline $\mathrm{L}$ & PI20 & 17 & 33 \\
$\mathrm{~L}$ & $\mathrm{FLA2}$ & 17 & 33 \\
$\mathrm{~S}$ & $\mathrm{PI} 20$ & 100 & 100 \\
$\mathrm{~S}$ & $\mathrm{FLA2}$ & 100 & 100 \\
O6 & PI20 & 50 & 100 \\
O6 & FLA2 & 0 & 100 \\
O24 & PI20 & 17 & 100 \\
O24 & FLA2 & 0 & 0 \\
\hline
\end{tabular}

Notes: Data are given as a percentage of treatment replicates (individual agar blocks) showing growth of surviving cysts $(n=6)$.

Abbreviations: L, large lamp; S, small lamp; O6 and O24, MPS OptiFree ${ }^{\circledR}$ Express for 6 hours and 24 hours. 
Table 2 Effect of UV irradiation on two strains of Acanthamoeba (PI20, FLA2) in amoeba saline

\begin{tabular}{|c|c|c|c|c|c|c|c|}
\hline \multirow[t]{2}{*}{ UV Source } & \multirow[t]{2}{*}{ Strain } & \multicolumn{6}{|c|}{ Treatment time (minutes) } \\
\hline & & 1 & 2 & 3 & 4 & 7 & 10 \\
\hline L & $\mathrm{PI} 20$ & $80(100)$ & $80(68)$ & $50(50)$ & $0(33)$ & $0(0)$ & $0(0)$ \\
\hline L & FLA2 & $90(100)$ & $60(50)$ & $40(50)$ & $0(33)$ & $0(0)$ & $0(0)$ \\
\hline S & $\mathrm{PI} 20$ & $100(100)$ & $100(83)$ & $100(100)$ & $90(100)$ & ND & $50(67)$ \\
\hline S & FLA2 & $100(100)$ & $90(100)$ & $100(100)$ & $90(100)$ & ND & $33(50)$ \\
\hline
\end{tabular}

Notes: Data are given as a percentage of treatment replicates (individual agar blocks) showing growth of surviving trophic amoebae and cysts (in parentheses) that survived treatment $(n=6$ or 10$)$.

Abbreviations: L, large UV light; S, small UV light; ND, no data.

some cysts survived in all the P120 cyst trials (ie, all the replicates yielded some survivors). This differential survival suggests that the action of the MPS depends on penetration across different cyst walls (reflecting different structures).

The effect of combining MPS disinfection with UV irradiation from the larger lamp is shown in Table 4. To kill trophic amoebae of both strains with MPS alone required 6 hours treatment (Table 3 ) but by combining with UV treatment for 3 minutes, all trophozoites were killed after just 1 hour in MPS. Indeed, even a short 1-minute UV treatment markedly reduced all trophic amoebae even with a short MPS treatment. The unexpected results showing $17 \%$ survival (equals 1 agar block of 6 in total) indicate that there were a few survivors after treatment, enough to grow up when sub-cultured on to fresh medium. Although the absolute number of surviving cells is unknown, it is likely that the prevalence of zero grow out in the replicates suggests that most of the approximate 300 to 500 cells challenged during the course of each experimental run (50 cells per replicate) were inactivated. It is also possible that the agar block contained a lone cyst that was not observed when setting up the experimental runs. Such a condition would lead to occasional unexpected survival. Cysts proved harder to kill although FLA2 could be killed when exposed to MPS for 24 hours alone. The pertinent data is the resistant strain P120 that survived $100 \%$ in 24-hour MPS (Table 3). With UV treatment, a short 1-minute exposure followed by 24 hours in MPS inactivated all cysts on two-thirds of the agar blocks (ie, at least 330 of the 500 cysts challenged were inactivated) while a 4-minute UV treatment left a maximum of 85 surviving cysts ( $17 \%$ survival, Table 4$)$. Even a 2- to 4-minute UV treatment followed by MPS treatment for 3 or 6 hours (a protocol that better mimics actual lens care) reduced cyst survival by approximately $50 \%$ (data range $17 \%-67 \%$ survival).

Not surprisingly, the small unit was less effective than the large UV unit at killing or inactivating cysts. FLA2 and P120 trophozoites were killed in MPS alone after 6 hours
(Table 3). With combined treatment (MPS and small lamp) all were killed after 3 hours in MPS with at least 1 minute of UV treatment (except for remnant survival of 17\% after 2 and 4 minutes) (Table 5). After 24 hours in MPS, P120 cysts were moderately reduced by $17 \%$ with 4 minutes UV treatment (relative to no reduction with MPS alone).

In the course of the grow-out experiments to test for surviving acanthamoebae on the surface of the agar blocks, rinsed blocks were placed amoeba side down on to fresh E. coli prey on new NNA plates. Surviving cells replicated after a lag phase and migrated as they consumed prey bacteria and grew (this constituted a positive result). The length of the lag phase (before the onset of migration that mimicked a growth curve) was noted in each experiment. Any delays in the lag phase were an indication of sub-lethal effects of treatment. The average lag phase for an exponentially growing culture from one of the controls was 1.1 days (standard deviation $[\mathrm{SD}]=0.3, \mathrm{n}=10$ ). In contrast, UV irradiation (small or large lamp) that did not kill all amoebae nonetheless did extend the lag phase, suggesting either cells were stressed or some were inactivated. For example, 1 minutes treatment on trophic amoebae with the small and large UV lamps extended the lag phase to 5.0 days \pm 3.5 and 3.5 days \pm 0.7 , respectively ( $\mathrm{n}=10$ for both treatments). There was less of a quantitative effect on cysts, even with the large hand held lamp. The control lag phase from 50 cysts inoculated was 3.0 days $\pm 0.1 \mathrm{SD}$, but this was only extended

Table 3 Effect of MPS (OptiFree ${ }^{\circledR}$ Express) on two strains of Acanthamoeba (PI20, FLA2)

\begin{tabular}{llllll}
\hline Strain & \multicolumn{5}{l}{ Treatment time (hours) } \\
\cline { 2 - 6 } & $\mathbf{0}$ & $\mathbf{I}$ & $\mathbf{3}$ & $\mathbf{6}$ & $\mathbf{2 4}$ \\
\hline $\mathrm{PI} 20$ & $100(100)$ & $20(\mathrm{ND})$ & $10(100)$ & $0(100)$ & $0(100)$ \\
FLA2 & $100(100)$ & $20(\mathrm{ND})$ & $10(100)$ & $0(100)$ & $0(0)$ \\
\hline
\end{tabular}

Notes: Data are given as a percentage of treatment replicates (individual agar blocks) showing growth of amoebae and cysts (in parentheses) $(n=6$ or I0). Abbreviation: ND, no data. 
Table 4 Effect of MPS (OptiFree ${ }^{\circledR}$ Express) and UV irradiation (large lamp) on two strains of Acanthamoeba (PI20, FLA2)

\begin{tabular}{|c|c|c|c|c|c|}
\hline \multirow{2}{*}{$\begin{array}{l}\text { MPS } \\
\text { (hours) }\end{array}$} & \multirow[t]{2}{*}{ Strain } & \multicolumn{4}{|c|}{ UV treatment time (minutes) } \\
\hline & & $\mathbf{I}$ & 2 & 3 & 4 \\
\hline I & $\mathrm{PI} 20$ & 0 (ND) & 0 (ND) & $0(N D)$ & $0(N D)$ \\
\hline I & FLA2 & 17 (ND) & 0 (ND) & 0 (ND) & 0 (ND) \\
\hline 3 & $\mathrm{PI} 20$ & $17(100)$ & $0(50)$ & $0(50)$ & $17(17)$ \\
\hline 3 & FLA2 & $0(100)$ & $17(67)$ & $0(50)$ & $0(50)$ \\
\hline 6 & $\mathrm{PI} 20$ & $0(100)$ & $0(67)$ & $0(50)$ & $0(33)$ \\
\hline 6 & FLA2 & $0(100)$ & $0(50)$ & $0(50)$ & $0(33)$ \\
\hline 24 & $\mathrm{PI} 20$ & $0(33)$ & $0(33)$ & $0(33)$ & $0(17)$ \\
\hline 24 & FLA2 & $0(0)$ & $0(0)$ & $0(0)$ & $0(0)$ \\
\hline
\end{tabular}

Notes: Data are given as a percentage of treatment replicates (individual agar blocks) showing growth of surviving trophic amoebae and cysts (in parentheses) $(\mathrm{n}=6$ or 10). UV treatment followed treatment with MPS for I, 3, 6 or 24 hours.

Abbreviation: ND, no data.

to 4.0 days $\pm 0.8 \mathrm{SD}$ after 3 minutes UV irradiation. In short, this effect indicates that many, even most amoebae as trophozoites or cysts on a treated agar block may have been killed, but if the few survivors grew out, the plate would have been scored as "positive", with apparent $100 \%$ survival. The inactivation data presented here, therefore, should be considered as conservative, ie, they likely underestimate killing (inactivation) of amoebae.

\section{Discussion}

To the best of our knowledge, this study is the first to investigate the effectiveness of a combined treatment to limit the growth of Acanthamoeba on contact lenses. The need for effective disinfecting treatments is clear. The recent investigations of $\mathrm{AK}$ outbreaks have demonstrated the importance of including Acanthamoeba as a test organism in the development and FDA (Food and Drug Administration) certification process of multipurpose contact lens disinfecting solutions. The CDC (Center for Disease Control) recently conducted a series of hearings and workshops and has reported on the need to develop standardized pre-market MPS testing procedures that will include Acanthamoeba. ${ }^{7}$

One of the ongoing problems for testing the efficacy of MPS and/or UV systems against eukaryotic microorganisms is the absence of standard protocols. Freely suspended amoebae represent a different challenge when compared with cells attached to a surface. Shoff and colleagues ${ }^{2}$ tested amoebae attached to an agar surface arguing that this method best mirrored cells embedded in a biofilm on a contact lens or in an accumulated biofilm layer in a lens case. Caroline and Campbell ${ }^{8}$ demonstrated that the lens case biofilm may be the greatest source of microbial contamination in lens cleaning systems. In the study by Shoff and colleagues, ${ }^{2}$ three popular multipurpose contact lens disinfecting solutions showed variable results, but also demonstrated that no MPS effectively killed the Acanthamoeba cysts of any strain tested.

The test method employed here only detected 100\% kill or inactivation within treatment replicates (individual agar blocks) and could not differentiate between survival of 1 or 50 amoebae on treatment replicates that grew out. Even so, the method is useful since it is rapid and gives minimal disturbance to amoebae being tested; they remain attached amid bacterial prey. While there was no attempt to estimate the number of cells killed in a test, a high proportion of 'zero survival' scores among replicates suggests that the treatment was indeed killing cells, even in those replicates that grew out. This is underscored by the lag phase data that detected sub-lethal effects. Treatments invariably increased the lag

Table 5 Effect of MPS (OptiFree ${ }^{\circledR}$ Express) and UV irradiation (small lamp) on two strains of Acanthamoeba (PI20, FLA2)

\begin{tabular}{|c|c|c|c|c|c|}
\hline \multirow[t]{2}{*}{ MPS (hours) } & \multirow[t]{2}{*}{ Strain } & \multicolumn{4}{|c|}{ UV treatment time (minutes) } \\
\hline & & $\mathbf{I}$ & 2 & 3 & 4 \\
\hline $\mathrm{I}$ & $\mathrm{PI} 20$ & 0 (ND) & 0 (ND) & 0 (ND) & 0 (ND) \\
\hline I & FLA2 & 17 (ND) & 33 (ND) & 17 (ND) & 17 (ND) \\
\hline 3 & $\mathrm{PI} 20$ & $0(100)$ & $0(100)$ & $0(100)$ & $17(100)$ \\
\hline 3 & FLA2 & $0(100)$ & $17(100)$ & $0(100)$ & $0(100)$ \\
\hline 6 & $\mathrm{PI} 20$ & $0(100)$ & $0(100)$ & $0(100)$ & $0(100)$ \\
\hline 6 & FLA2 & $0(100)$ & $0(100)$ & $0(83)$ & $0(100)$ \\
\hline 24 & $\mathrm{PI} 20$ & $0(100)$ & $0(83)$ & $0(83)$ & $0(83)$ \\
\hline 24 & FLA2 & $0(0)$ & $0(0)$ & $0(0)$ & $0(0)$ \\
\hline
\end{tabular}

Notes: Data are given as a percentage of treatment replicates (individual agar blocks) showing growth of surviving trophic amoebae and cysts (in parentheses) ( $\mathrm{n}=6$ or 10 ). UV treatment followed treatment with MPS for I, 3, 6 or 24 hours. Abbreviation: ND, no data. 
phase relative to controls, indicating that surviving cells were either reduced and/or stressed.

Also relevant to testing protocols is the variation in strain susceptibility highlighted in the current study. Strain FLA2 (T4) was far more sensitive to treatment than P120, the T3 genotype. This result agrees with the study by Shoff and colleagues $^{2}$ who concluded that genotypes T3 and T5 were harder to kill than T4 types, and may be better strains for future testing. These differences are probably attributable to cyst wall structural differences that affect movement of MPS into the cell. Interestingly, UV treatment inactivated cysts of both strains regardless of cyst wall structure. Likewise, the present study reinforced the importance of cyst age as a factor in testing. ${ }^{6}$ Mature cysts were more resistant to treatment than young cysts, and mature cysts, from a resistant genotype (T3), would be a prudent choice for future testing.

Ultraviolet lamps are known for their germicidal effectiveness and are employed in many industrial applications. However, these are considerably more powerful and therefore dangerous to the operator than the relatively modest power units employed here. Low dose UV irradiation may alleviate the problem of long-term exposure of contact lenses to UV irradiation. Dolmand and Dobrogowski ${ }^{9}$ found softening and opacification of lenses when using a $253.7 \mathrm{~nm}$ light with an intensity of $1100 \mathrm{~W} / \mathrm{cm}^{2}$.

One commercially available lens cleaning system is the PuriLens ${ }^{\circledR}$ contact lens cleaning system and although this system inactivates or kills ocular bacterial pathogens, it was unable to kill Acanthamoeba cysts in a single run. ${ }^{10}$ Importantly, during an evaluation of the PuriLens ${ }^{\circledR}$ contact lens care system, Choate and colleagues ${ }^{11}$ reported that lenses were not affected by UV irradiation after 180 cleaning cycles. In light of the above, the present study set out to look for synergy between a popular MPS (OptiFree ${ }^{\circledR}$ Express) and two inexpensive UV systems to determine whether irradiation from these relatively weak light sources could enhance the effectiveness of the MPS.

While the study is investigational only and not intended to certify a contact lens home cleaning method, it is noteworthy that if a contact lens wearer used precautions that come with these hand held units, there may be a benefit to a UV irradiation pretreatment prior to MPS disinfection treatment. While the small, battery powered unit in combination with MPS failed to kill all cysts within 4 minutes, it did reduce survival to $83 \%$ for the resistant genotype T3. This compares to $100 \%$ survival in MPS alone. It is also worth noting that many more cells might have been inactivated by the treatment since the method used does not account for differential killing. Moreover, the treatment of young cysts was not tested using
MPS and UV but these would be easier to kill as reflected in the initial trials reported in Table 1. Lastly, while current ISO standards do not call for the testing of MPS against Acanthamoeba, Schatz and colleagues ${ }^{12}$ demonstrated the efficacy of a rub step in reducing microbial populations on contact lenses and Seal and colleagues ${ }^{13}$ and later Butcko et $\mathrm{al}^{14}$ showed that the inclusion of a rub step resulted in the elimination of Acanthamoeba contamination of contact lenses. Clearly there are many variables still to be addressed before a method of treatment can be advocated using both physical and chemical treatment. However, the data presented here point the way towards future work that could produce a set of protocols for effectively limiting the contamination of contact lenses and lens cases with Acanthamoeba and consequently reduce the incidence of $\mathrm{AK}$.

\section{Acknowledgments}

The authors are indebted to Dr Fred Dobbs, Old Dominion University, Norfolk, VA, USA, for his helpful comments throughout this study.

\section{Disclosures}

The authors disclose no conflicts of interest.

\section{References}

1. Hiti K, Walochnik J, Faschinger C, et al. One- and two-step hydrogen peroxide contact lens disinfection solutions against Acanthamoeba: how effective are they? Eye. 2005;19:1301-1305.

2. Shoff ME, Rogerson A, Shatz S, Seal DV. Variable responses of Acanthamoeba strains to three multipurpose lens cleaning solutions. Optom Vis Sci. 2007;84:202-207.

3. Page FC. A New Key to Freshwater and Soil Gymnamoebae. Freshwater Biological Association, Cumbria, UK; 1988:122.

4. Booton GC, Rogerson A, Bonilla TD, et al. Molecular and physiological evaluation of subtropical environmental isolates of Acanthamoeba spp., causal agent of Acanthamoeba keratitis. J Eukaryot Microbiol. 2004;51:192-200.

5. Buck SL, Rosenthal RA, Schlech BA. Methods used to evaluate the effectiveness of contact lens care solutions and other compounds against Acanthamoeba: a review of the literature. CLAO J. 2000;26:72-84.

6. Kilvington S, Anger C. A comparion of cyst age and assay method of the efficacy of contact lens disinfectants against Acanthamoeba. Br J Ophthalomol. 2001;85:336-340.

7. Verani JR, Lorick SA, Yoder JS, Beach MJ, Braden CR, Roberts JM. National outbreak of Acanthamoeba keratitis associated with use of a contact lens solution, United States. Emerg Infect Dis Aug. Available (2009) from http://www.cdc.gov/EID/15/8/1236.htm

8. Caroline PJ, Campbell RC. Strategies of microbial cell survival in contact lens cases. Contact Lens Forum. 1990;15:27-32.

9. Dolman PJ, Dobrogowski MJ. Contact lens disinfection by ultraviolet light. American. J Ophthamol. 1989;108:665-669.

10. Hwang TS, Hyon JY, Song JK, Reviglio VE, Spahr HT, O’Brien TP. disinfection capacity of PuriLens contact lens cleaning unit against Acanthamoeba. Eye Contact Lens. 2004;30:42-43.

11. Choate W, Fontana F, Potter J, Schachet J, et al. Evaluation of the PuriLens ${ }^{\circledR}$ contact lens care system incorporating UV disinfection and hydrodynamic shear cleaning. CLAO J. 2000;26:134-140. 
12. Schatz S, Manzella C, Wenger C, Darius D, Laubach H. Comparion of Complete Easy Rub with OptiFree Replenish in contact lens hygiene. Optom Vis Sci. 2008:Abstract 85060.

13. Seal DV, Dalton A, Doris D. Disinfection of contact lenses without tap water rinsing: Is it effective? Eye. 1999;13:226-230.
14. Butcko V, McMahaon TT, Joslin CE, Jones L. Microbial keratitis and the role of rub and rinsing. Eye Contact Lens. 2007;33:421-425.
Clinical Optometry

\section{Publish your work in this journal}

Clinical Optometry is an international, peer-reviewed, open access journal publishing original research, basic science, clinical and epidemiological studies, reviews and evaluations on clinical optometry. All aspects of patient care are addressed within the journal as well as the practice of optometry including economic and business analyses. Basic and clinical

Submit your manuscript here: http://www.dovepress.com/clinical-optometry-journal

\section{Dovepress}

research papers are published that cover all aspects of optics, refraction and its application to the theory and practice of optometry. The manuscript management system is completely online and includes a very quick and fair peer-review system, which is all easy to use. Visit http://www.dovepress. com/testimonials.php to read real quotes from published authors. 